\title{
MODELING AND MEASUREMENT OF RESIDUAL STRESSES IN A FORGED IN718 SUPERALLOY DISC
}

\author{
D. Dye ${ }^{1}$, B.A. Roder ${ }^{2}$, S. Tin ${ }^{2}$, M.A. Rist ${ }^{3}$, J.A. James ${ }^{3}$ and M. R. Daymond ${ }^{4}$ \\ ${ }^{1}$ Department of Materials, Royal School of Mines, Imperial College, Prince Consort Road, South Kensington, London SW7 2BP, UK \\ ${ }^{2}$ Rolls-Royce University Technology Partnership, University of Cambridge, Department of Materials Science and Metallurgy, \\ Cambridge CB2 3QZ, UK \\ ${ }^{3}$ Open University, Department of Materials Engineering, Milton Keynes MK7 6AA, UK \\ ${ }^{4}$ ISIS, Rutherford Appleton Laboratory, Chilton, Didcot OX11 0QX, UK
}

Keywords: quenching, residual stress, forgings, neutron diffraction

\begin{abstract}
The residual stresses present in a quenched IN718 aeroengine compressor disc forging have been characterized using neutron diffraction and the results compared to those obtained from a finite element (FE) model for the quenching process. The $\sim 40 \mathrm{~kg}$ forging had a diameter of $\sim 400 \mathrm{~mm}$ and a maximum thickness of $\sim 45 \mathrm{~mm}$. Neutron path lengths of up to $60 \mathrm{~mm}$ were required to record strain components at the deepest points within the material. The residual hoop and radial stresses measured are generally compressive at the surface, up to $600 \mathrm{MPa}$ and tensile at depth, up to $400 \mathrm{MPa}$, whilst the radial stresses are generally small. The deviatoric stresses are generally $<150 \mathrm{MPa}$. FE model predictions of the residual stress are reasonable agreement with the measured values.
\end{abstract}

\section{Introduction}

The production of Ni-base superalloy disc forgings is a complex process involving many stages. The microstructural response of the material during forging, quenching and heat-treatment governs the resultant mechanical properties and residual stresses. After forging, a solution heat treatment followed by rapid oil or water quenching is used. During the quench, residual stresses arise due to non-uniform thermal contraction and (possibly) local transformation-induced shrinkage. The component is then machined into its final shape by turning on a lathe, a process that removes around $60 \%$ of the material. Subsequent to final joining, stress relief and aging heat treatment are used to develop the final microstructure, precipitate the intermetallic phases $\left(\gamma^{\prime \prime}\right.$ and $\left.\gamma^{\prime}\right)$ and enhance the strength of the alloy.

During machining, residual stresses within the disc due to quenching can redistribute and cause a physical distortion of the component. The resulting movement could potentially lead to failure of the finished component as the dimensional tolerances become altered. In severe cases, dimensional instability (buckling) can occur. This is particularly the case where intricate shapes are produced, such as drive arms and webs. For this reason, it is desirable to model the machining sequence to optimize the process parameters and avoid these problems. In addition, if the amount of material to be removed can be minimized, the forging operations can be performed using smaller, less costly equipment and savings in material can be achieved. An obvious precondition of such modeling is knowledge of the magnitude and nature of the residual stresses distributed within the component. In addition to the manufacturing issues, residual stresses may potentially influence the fatigue performance and subsequent service life of the finished component $[1,2]$.

Manufacturers seek to predict and minimize this instability, but the understanding of microstructures in forged components and the design of manufacturing sequences have traditionally been reliant upon iterative workshop trials and the experience of forging metallurgists. Industry is increasingly recognizing the benefits of computer simulation and there is a drive to implement Finite Element Analysis (FEA) techniques to predict the properties of the final component from the processing parameters, such that integrated optimization of the entire process route can be performed.

Most of the available techniques for the measurement of residual stresses in such thick components, such as x-ray diffraction or hole-drilling are limited to the measurement of surface or near-surface $(<1 \mathrm{~mm})$ stresses. Even neutron diffraction, which derives its penetrating power from the weak interaction of neutrons with matter, has usually been limited to depths of $\sim 25 \mathrm{~mm}$. However, new instruments such as SMARTS at Los Alamos and ENGIN-X at ISIS have been built in the last few years specifically for stress measurement, and these are capable of measuring stresses at greater depths.

In this paper, a series of through thickness residual stress measurements made in a full-scale, high pressure compressor disc forging are compared to predictions from a finite element quench model [3]. The magnitude and character of the residual stresses within the solutioned and quenched IN718 forging were rationalized on the basis of these results. 


\section{Experiment}

Neutron diffraction measurements were conducted on a full-size compressor disc (rotor) forging of IN718 supplied by Wyman-Gordon (Livingston, Scotland). Following a standard solution heat-treatment, the disc was rapidly cooled via a water fog. Microstructures of the as-quenched forging were analyzed and revealed to consist of fine, equiaxed single-phase $\gamma$ grains $(\mathrm{d}=\sim 7 \mu \mathrm{m})$ and a small fraction of $\delta$ precipitates distributed along the grain boundaries, Figure 1. Neutron diffraction measurements were then made using the ENGIN-X instrument at the ISIS facility in the Rutherford Appleton Laboratories (RAL) in Oxfordshire, UK. ISIS is a spallation neutron source, that is, pulses of neutrons are produced at regular intervals by proton bombardment of a high-z material, lightly moderated and then distance-collimated by energy using the time of flight to the detector. The advantage of this approach is that the entire diffraction pattern is obtained and that each detector is naturally making a measurement that is radial in reciprocal space - that is, it is a true measurement of the lattice parameter $d$.

Neutron path lengths of up to $60 \mathrm{~mm}$ were required to obtain diffraction information from the deepest locations within the material. Neutron diffraction strain measurement uses the change in lattice spacing to infer the elastic strain in the material, in effect using the lattice expansion as a microscopic internal strain gauge. The strain of a given $\{h k l\}$ peak is obtained from the change in lattice spacing $d$ as follows.

$$
\varepsilon_{h k l}=\frac{\Delta d}{d_{0}}
$$

Measurements were made selectively using grains in each diffraction orientation, that is with their $\{h k l\}$ planes normal to the radial, hoop and transverse directions. Measurements of the strain free reference $d_{0}$ were made from samples cut from the disc by electro-discharge machining (EDM).

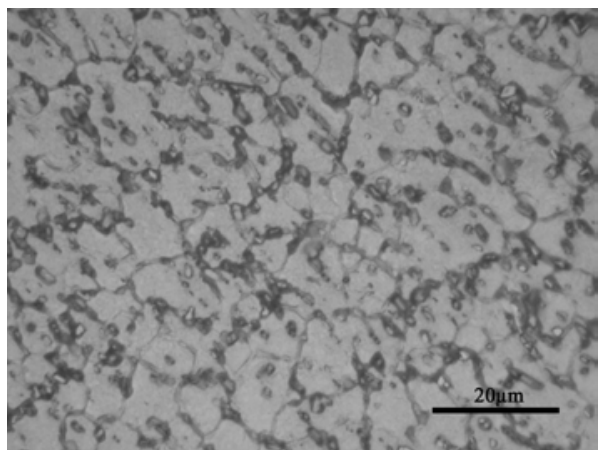

Figure 1: Microstructure of the as-quenched IN718 forging showing grain boundary $\delta$ and equiaxed grains of $\gamma$.
The major complicating factor associated with the diffraction strain measurement approach is caused by anisotropy in the elastic and plastic response of grains in different orientations, and the peak overlap associated with a multicomponent microstructure comprised of $\gamma, \delta, \gamma^{\prime}$ and $\gamma^{\prime \prime}$. However, a number of studies [4,5] have shown that for IN718 it is reasonable to simply apply a Reitveld fit of a single lattice parameter $a$ to the entire diffraction spectrum obtained and then to use the bulk engineering elastic constants for the conversion of the measured lattice strains to macroscopic stress. These assumptions were consistent with the microstructure of the as-quenched forging.

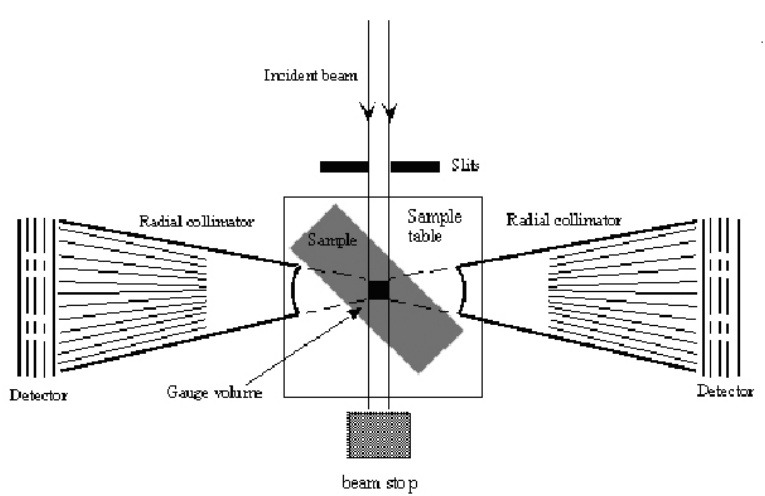

Figure 2: General Arrangement of the ENGIN-X diffractometer at ISIS, illustrating the illumination of a gauge volume.

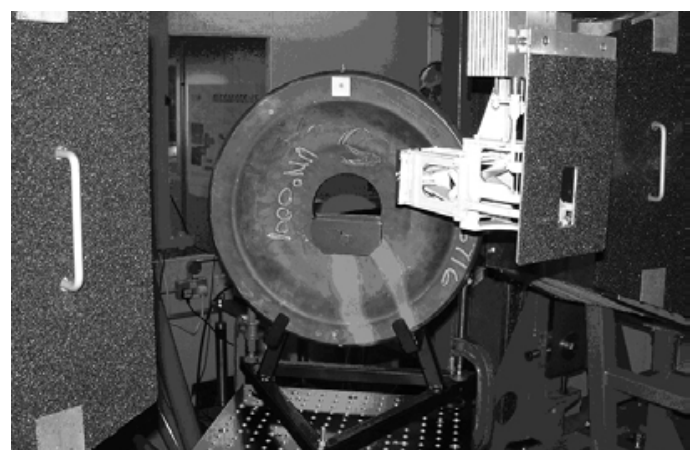

Figure 3: Arrangement of the sample of the diffractometer for measurements of the hoop and axial strain.

A key consideration in making a diffraction measurement is the sampling volume used in the experiments, which is obtained by masking the beam and detector, Figure 2. In order to obtain the measurements of strain from locations deep within the component in a reasonable time, and to avoid variations due to sampling of a small number of grains, the measurements were made from gauge volumes between $4 \times 4 \times 4$ and $4 \times 4 \times 10 \mathrm{~mm}$. The arrangement of the sample on the sample table is shown in Figure 3, and the program of measurement locations in Figure 4. Measurements of axial and hoop strain were made along a vertical section, with the disc mounted as shown in 


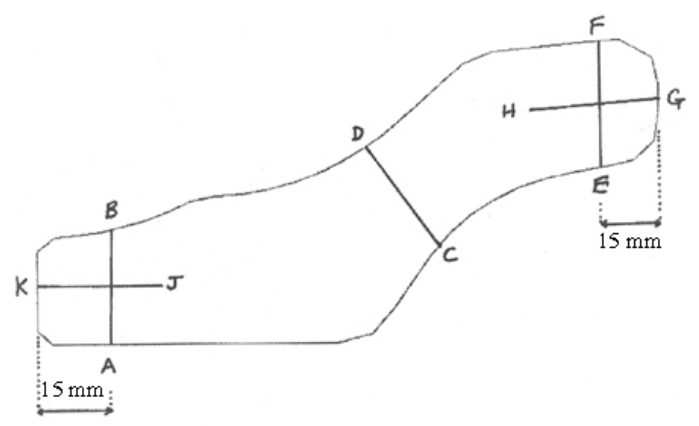

Figure 4: Schematic of the strain scanning measurements made in the disc. The inner radius of the disc at point $\mathrm{K}$ is approximately $50 \mathrm{~mm}$ from the line of symmetry.

Figure 3, and of axial and radial strain along a horizontal section. Symmetry was assumed in the stresses around the disc, and this assumption verified by comparing the axial strains measured from both locations; hence a complete (principal) strain tensor could be determined, assuming that the measurements were made in the directions of principal strain. This assumption will be tested using the model results that follow.

\section{Modeling}

A 2D axisymmetric sequentially coupled thermalmechanical finite element model of the quenching process was constructed using a mesh generated using the IDEA-S package and ABAQUS as the solver. Linear elements were used for the thermal analysis (type DCAX4) and linear elements with enhanced bending modes for the mechanical analysis (type CAX4I). Compatibility of the temperature field with the displacement field in the mechanical model was guaranteed using the interpolation routine incorporated into ABAQUS, as discussed in previous work [6]. The mesh was constructed to represent a disc with the machined center hole. Hexahedral elements approximately $1 \mathrm{~mm}$ in length in both the radial and axial direction were used; the mesh is shown in Figure 5.

The material properties used were those appropriate for solution heat treated IN718 and previously assessed from the literature and experimentation $[6,7,8]$, Table 1 . The temperature-dependent surface cooling coefficients $h$, Figure 6 , were obtained previously $[3,9]$ by using the measured temperature profile of an immersion-quenched IN718 rod as a boundary condition in a finite element model, where the thermal flux $q$ is related to the temperature difference between the water and the surface $\Delta T$ by $q=h \Delta T$. In the model, an initial temperature of $970{ }^{\circ} \mathrm{C}$ was used at the initiation of cooling, representative of the temperatures applied to solution heat treatment, and the sink (water) temperature was assumed to be $20^{\circ} \mathrm{C}$.

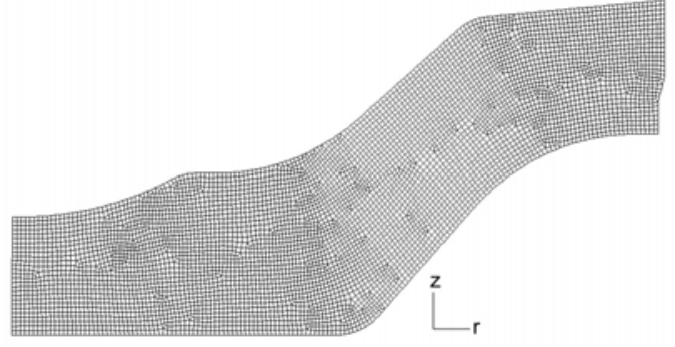

Figure 5: Mesh used in the analyses. The element length is approximately $1 \mathrm{~mm}$.

The yield strength $\sigma_{\mathrm{y}}$ and constitutive model require more careful consideration. In line with the literature [10], a rate-independent, isotropic linear hardening model was assumed, setting the hardening coefficient $d \sigma_{\mathrm{y}} / d \varepsilon_{\mathrm{p} 1}$ to $0.01 \mathrm{E}$. The temperature dependence of the yield strength was fitted to data for solution heat-treated IN718 [6,8], using the equation

$$
\sigma_{y}\{T\}=\frac{\sigma_{y}(\mathrm{RT})-\sigma_{y}(\mathrm{HT})}{1+\exp \{(T-b) / c\}}+\sigma_{y}(\mathrm{HT})
$$

where $\sigma_{\mathrm{y}}(\mathrm{RT})$ is the room-temperature yield strength and $\sigma_{\mathrm{y}}(\mathrm{HT})$ is the yield strength at high temperatures, above the $\gamma^{\prime}$ and $\gamma^{\prime \prime}$ solvii. The material constants $b$ and $c$ are determined via fitting of the equation to experimental data. The yield curve used is shown in Figure 7. It should be noted that this approach assumes that the temperature dependence of the yield curve does not depend on the kinetics of solute precipitation and takes no account of, for example, coarsening of the $\gamma^{\prime}$ and $\gamma^{\prime \prime}$ precipitates, which is known to have a strong effect on the yield strength of nickel-base superalloys [11]. However, in this particular case the thermal history of the forging upon quenching was unlikely to result in any significant precipitation/coarsening of the various intermetallic precipitates that influence the flow stress.

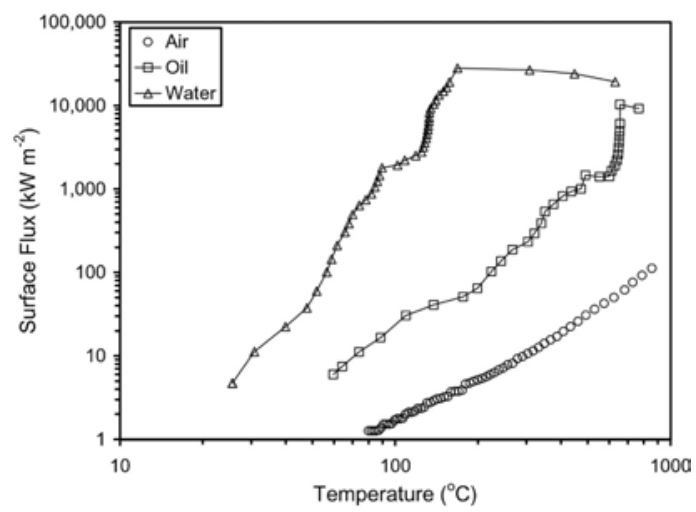

Figure 6: Film cooling coefficients used in the model for water quenching, after $[3,9]$. 
Table 1: Temperature-dependent thermal and mechanical properties used in the finite element models, derived from $[7,8]$. The thermal expansion $\varepsilon_{t h}$ is defined as the total expansion divided by the temperature, $\varepsilon_{t h}=\alpha T$, not the instantaneous expansion $\alpha=d \varepsilon_{t h} / d \mathrm{~T}$.

\begin{tabular}{|c|c|c|c|c|c|}
\hline $\begin{array}{c}\text { Temperature } \\
T \\
\left({ }^{\circ} \mathrm{C}\right)\end{array}$ & $\begin{array}{c}\text { Thermal } \\
\text { Conductivity } \\
\kappa \\
\left(\mathrm{Wm}^{-1} \mathrm{~K}^{-1}\right) \\
\end{array}$ & $\begin{array}{c}\text { Density } \\
\rho \\
(\mathrm{kgm}-3)\end{array}$ & $\begin{array}{c}\text { Specific Heat } \\
\text { Capacity } \\
C_{p} \\
\left(\mathrm{Jkg}^{-1} \mathrm{~K}^{-1}\right)\end{array}$ & $\begin{array}{l}\text { Mean Thermal } \\
\text { Expansion } \\
\text { Coefficient } \alpha \\
\left(10^{-6}\right)\end{array}$ & $\begin{array}{c}\text { Young's Modulus } \\
\text { E } \\
\text { (GPa) }\end{array}$ \\
\hline 0 & 11.02 & 8226 & 424 & 12.8 & 197 \\
\hline 100 & 12.75 & 8190 & 434 & 13.1 & 197 \\
\hline 200 & 14.36 & 8160 & 448 & 13.4 & 197 \\
\hline 300 & 15.96 & 8130 & 463 & 13.8 & 197 \\
\hline 400 & 17.60 & 8090 & 480 & 14.2 & 197 \\
\hline 500 & 19.18 & 8050 & 500 & 14.0 & 196 \\
\hline 600 & 20.77 & 8010 & 525 & 15.1 & 194 \\
\hline 700 & 22.36 & 7960 & 560 & 15.7 & 187 \\
\hline 800 & 23.95 & 7910 & 605 & 16.4 & 165 \\
\hline 850 & 24.53 & 7890 & 625 & 16.8 & 145 \\
\hline 900 & 25.10 & 7860 & 636 & 17.1 & 130 \\
\hline 1000 & 26.83 & 7810 & 645 & 17.5 & 105 \\
\hline
\end{tabular}

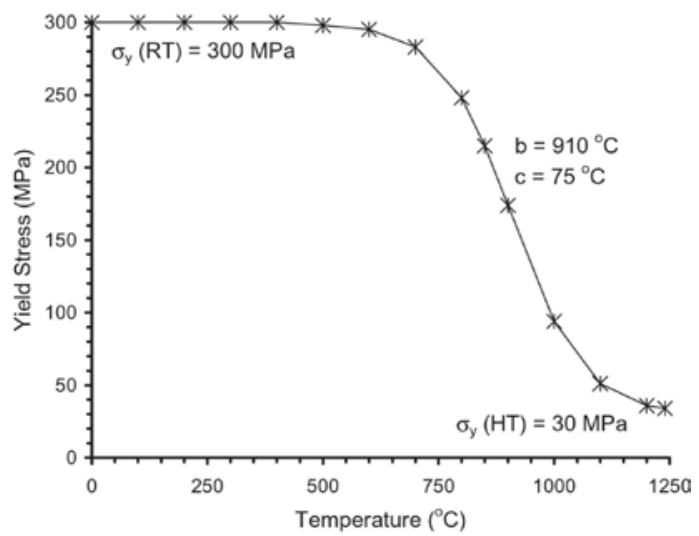

Figure 7: Variation in the yield stress used for solutionheat treated IN718, from $[3,7,8]$. The symbols refer to the equation in the text.

\section{Results and Discussion}

\section{$\underline{\text { Stress and Strain Measurements }}$}

Measurements of the full stress tensor were made along the two axial sections through the disc, one near the bore at $\mathrm{AB}$, and one at the rim $\mathrm{EF}$, Figure 8. For the remaining three sections measurements were made of only the hoop and axial strains, Figure 9. The uncertainties have been calculated from the uncertainty in the lattice parameter from the least-squares peak fitting package General Structure Analysis System (GSAS) [12]. The uncertainty in the elastic constants has been ignored as it is a systematic, rather than a random, error.

The major stresses are the hoop and radial stresses, with the axial stresses being smaller, within the range of $100 \mathrm{MPa}$ tensile to $200 \mathrm{MPa}$ compressive. The hoop and radial stresses are compressive at the surface, up to $600 \mathrm{MPa}$, and tensile in the core of the disc, up to $400 \mathrm{MPa}$.

The magnitude of the through thickness stress measurements at the disc rim (EF) are generally larger when compared to the stresses at the disc bore (AB). This is consistent with the trend that might be expected if one considers the situation where a cooling surface contracts over a hot core. In that case, tensile radial and hoop stresses will develop and result in the development of tensile plastic strains at the surface. When the entire disc has cooled down, these would then result in compressive stresses at the surface and tensile stresses in the core, which is observed in the measurements along $\mathrm{AB}, \mathrm{CD}$ and $\mathrm{KJ}$. Correspondingly, at the radial edges of the disc, a similar pattern would emerge, but in this case the cooling shell is constrained in the axial and hoop directions, but not the radial, and therefore the same pattern is developed but along different principal axes. Again, this is the pattern observed at the points $\mathrm{K}$ and $\mathrm{G}$.

Numerical Model

The temperature evolution predicted during quenching at the surface on the bore and rim and in the center of the disc, furthest removed from any surface, is shown in Figure 10. In addition, the temperature distribution $10 \mathrm{~s}$ into the quenching process is shown in Figure 11. Even in the center of the disc, the total time between $950{ }^{\circ} \mathrm{C}$ and $500{ }^{\circ} \mathrm{C}$ available for ageing is less than $40 \mathrm{~s}$. These cooling rates are sufficiently high to suppress the precipitation of $\gamma^{\prime \prime}$, resulting in minimal hardening of the material. The isotherm separation, and hence the quench rate, are almost directly proportional to the distance from the nearest free surface, as might be expected. 

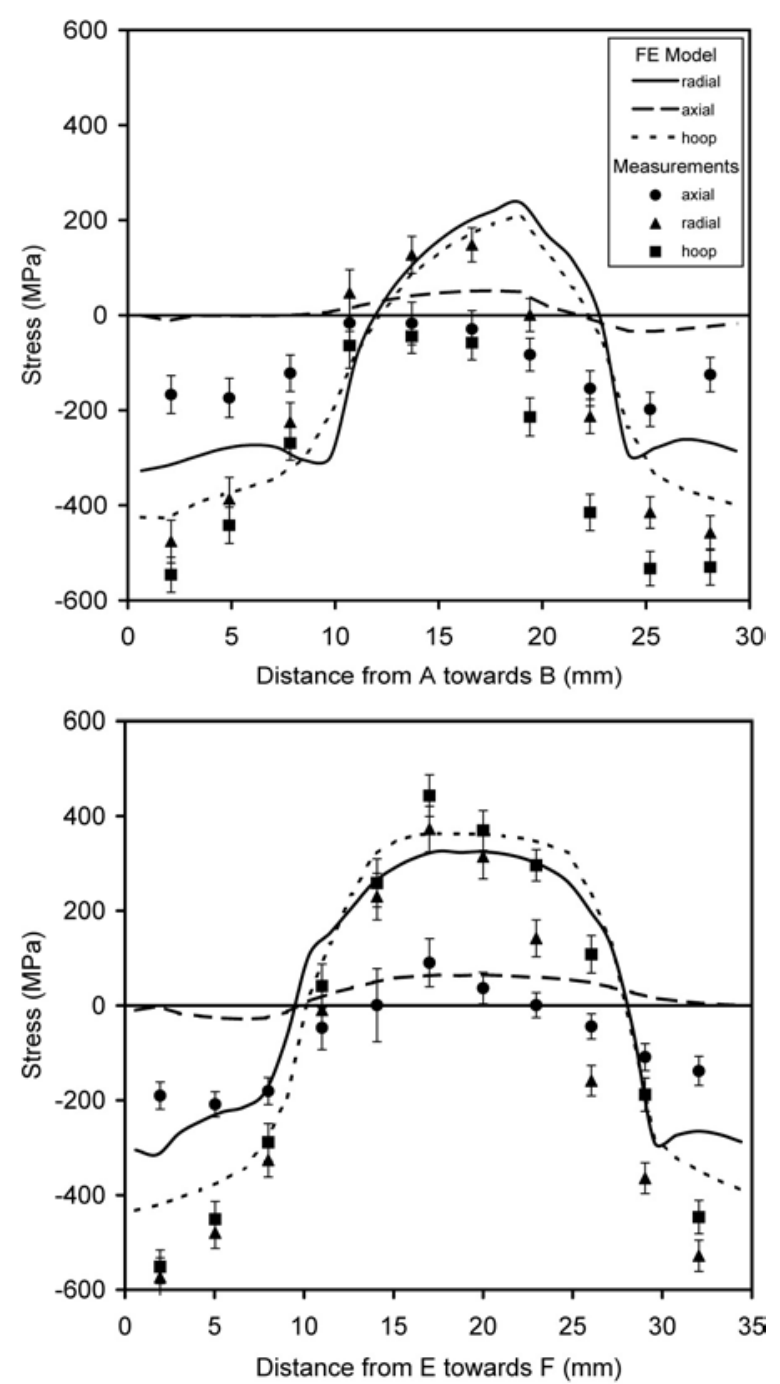

Figure 8: Measured and predicted stresses along axial sections near the bore $(\mathrm{AB})$ and $\operatorname{rim}(\mathrm{EF})$.

The consequent quenched residual stresses are shown in Figure 12, and confirm that the major stress components are the radial and hoop stress. All the predicted stress components appear balanced. The axial stresses occur in the constrained rim and bore region, as mentioned above, and in the inclined region at mid-width where the surface normal also has a significant radial component. The predicted shear stresses are of the same magnitude as the axial stresses, in the range 125 to $-175 \mathrm{MPa}$, and occur (i) sub-surface near the radial edges, a feature of 'doming' at the corners of the disc, and (ii) in the inclined region, which indicates that the disc is susceptible to bending. In addition, the presence of significant shear stresses may potentially influence the reliability of the stress measurements, which assumed that the shear stresses were zero, i.e that the principal axes were correctly identified a priori. However, this assumption is only violated along the scan $\mathrm{CD}$, which is a strain scan, not a stress measurement; the other scans pass through regions of low shear stress and will therefore be reliable.
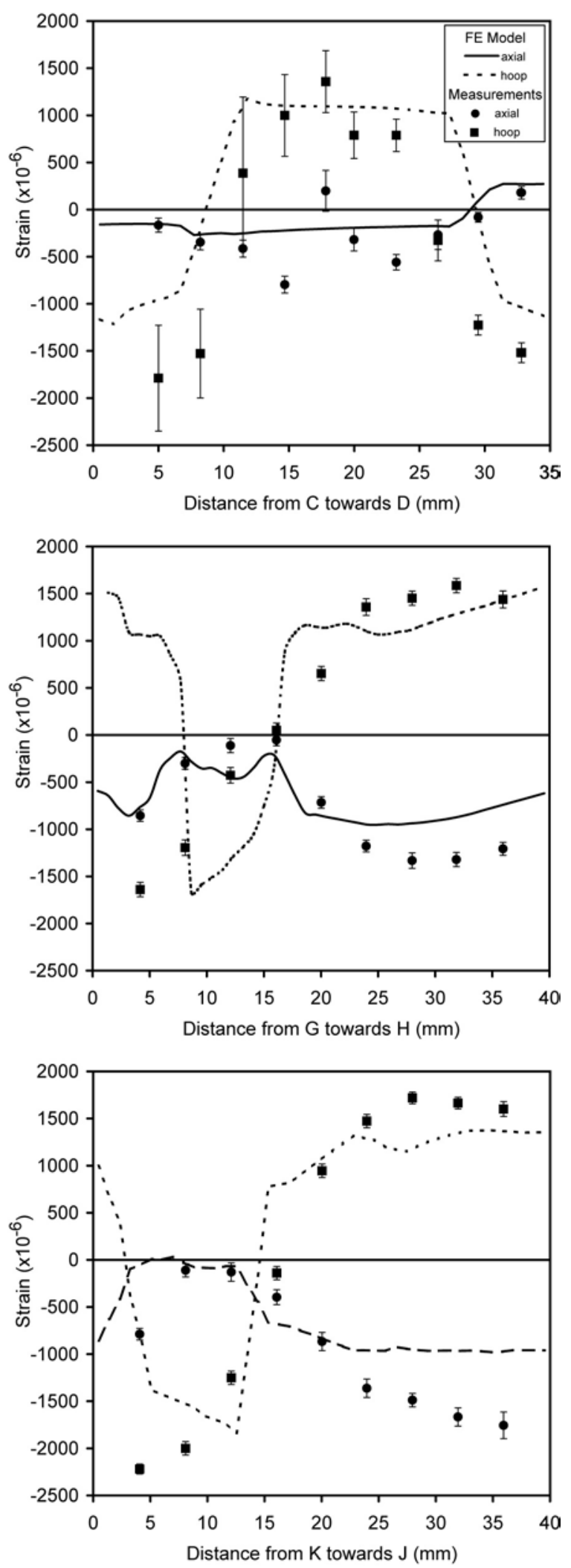

Figure 9: Measured and predicted strains along the three sections $\mathrm{CD}$, at mid-radius, $\mathrm{KJ}$, entering though the bore at mid-depth, and $\mathrm{GH}$, entering through the rim at mid-depth. In all the graphs, 0 is at the surface. 


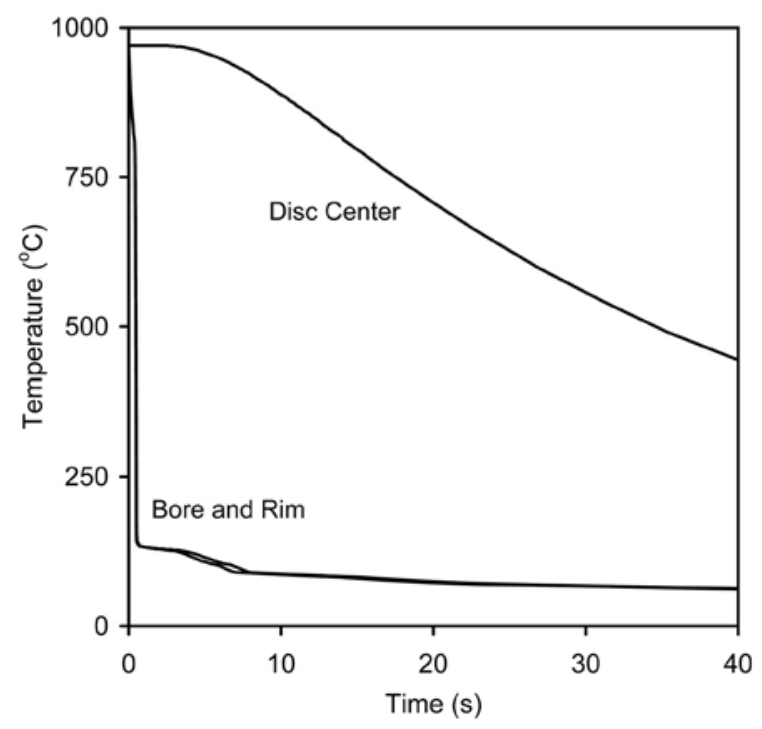

Figure 10: Evolution of the temperature at the surface of the disc in the rim and bore during quenching, and in the core of the disc.

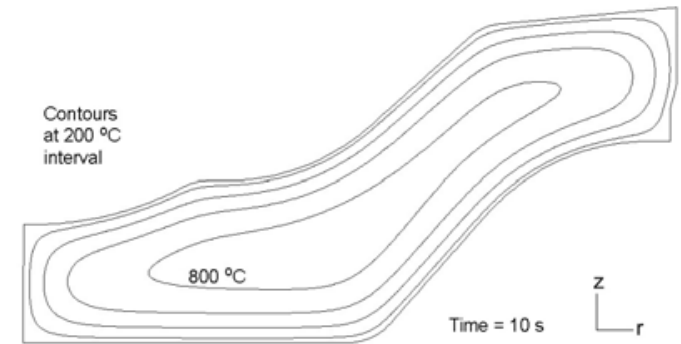

Figure 11: Transient temperature distribution during the quench sequence.

The dominant hoop stress is tensile in the core of the disc, as expected, and compressive on all the disc surfaces. It is of note that the regions of highest tensile stress lie not at the deepest points but are instead deflected towards the convex surfaces. These regions are exposed to relatively greater cooling, and therefore it might be expected that the plastic strains induced and therefore the final residual stresses are greater near these features.

The radial stresses are also significant, and are similarly dominated by tensile stresses in the deepest locations in the disc. The radial stresses fall to zero at the bore and rim, as required by equilibrium. Unlike the hoop stresses, the regions of greatest tensile stress are not deflected towards the convex surfaces, but the regions of greatest compressive stress are located at the flat and concave surfaces.

\section{Model - Measurement Comparison}

The model predictions (lines) and the measurements (points) are directly compared in Figures 8 and 9. The lines $\mathrm{GH}$ and KJ have the simplest stress profiles, similar to that of an infinitely long quenched bar, with tensile hoop strains and compressive axial strains in the interior of the disc. Discrepancies exist between the measured and predicted hoop and axial strains at regions near the surface. While the measured strain components are compressive in nature at regions near the surface of the forging, localized tensile strains are predicted from the model. Outside of the regions within $\sim 7 \mathrm{~mm}$ of the surface, the measured hoop and radial strains are similar in trend and magnitude to the predictions. The extent of the compressive region and the transition of the hoop strains to tensile in character along these segments are correctly predicted.

Along $\mathrm{CD}$, trend agreement between the measured and predicted strains is again observed, except for the highest uncertainty hoop strain measurements near C. The large uncertainty in these points is caused by the long path lengths and therefore reduced counting statistics. In view of the significant strain gradients occurring in this region, this agreement is felt to be extremely encouraging in terms of the feasibility of positioning a large component and measuring strains deep within it with a high degree of fidelity.

The two regions chosen for the full-tensor stress measurements, $\mathrm{AB}$ and $\mathrm{EF}$, correspond closely to the bore and rim of the machined disc, and therefore the depth of the stresses in this region are indicative of the amount of material that must be machined away before a strain and distortion free component can be obtained. In both cases, the predicted and measured stresses agree in trend and magnitude, although there are discrepancies. The measured compressive residual stresses at the surfaces A, B, E and F were greater than those predicted by more than $100 \mathrm{MPa}$. Considering the various parameters in the numerical model, these discrepancies are likely to be attributed to differences in yield strength between the modeled and actual material behavior. The yield stress of IN718 is known to vary from $300 \mathrm{MPa}$ to over $1200 \mathrm{MPa}$ depending on the grain size and heat treatment. In this case, the extremely fine grain size $(\sim 7 \mu \mathrm{m})$ present within the as-quenched forging likely contributed to the differences.

In addition, the measured hoop stresses across section $A B$ are uniformly compressive in nature while the hoop stresses at $\mathrm{EF}$ are comprised of both compressive and tensile components. Since a balance would be expected across both the $\mathrm{AB}$ and $\mathrm{EF}$ sections, it appears that a slight discrepancy exists between the measurements and model predictions. However, the strain measurements along $\mathrm{GH}$ and $\mathrm{KJ}$ are consistent with the nature of the stresses along $\mathrm{AB}$ and $\mathrm{EF}$ as they reveal that both the hoop and axial strain components approach zero near regions in which the residual stresses were measured. Finally, the measured profile in $\mathrm{AB}$ was skewed approximately $5 \mathrm{~mm}$ axially compared to the model results, which may be an effect of the insertion of the disc in the quench medium, which was 

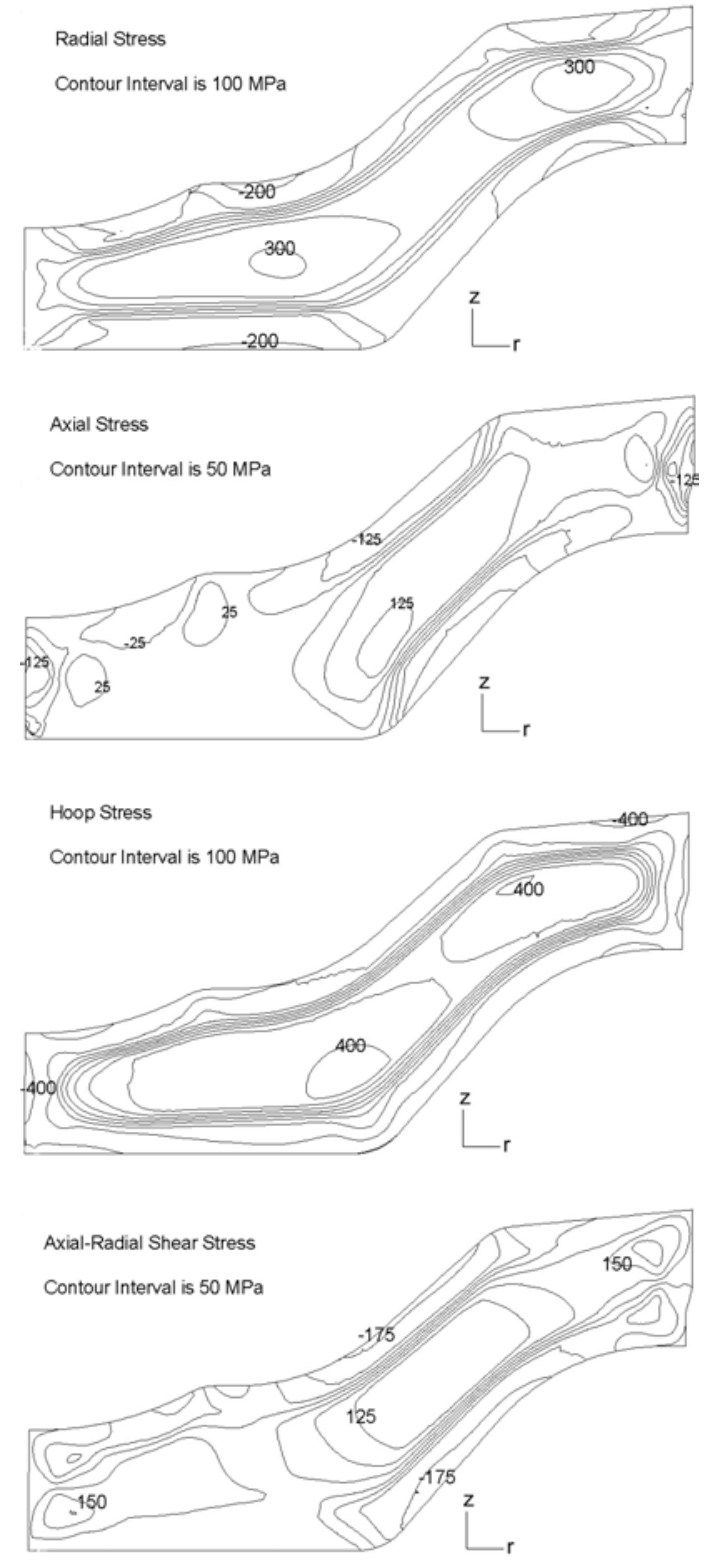

Figure 12: Predicted residual stresses in the disc after quenching.

not modeled. This effect, however, is not repeated in section EF close to the rim, which is in generally better agreement with the measurements.

Overall, the agreement between the experimental residual stress measurements and the preliminary, industrial model of the quench process can be regarded as satisfactory. Current experiments are specifically aimed at providing temperature dependent yield strengths specific to the asquenched microstructure in the forging. Coupling of this information with validated measurements of the thermal boundary conditions are likely to yield parameters that enable optimization of the FE model and provide better agreement with the measured values.

\section{Summary and Conclusions}

A numerical model for the water quenching process has been demonstrated in the case of the post-solutioning quench of an IN718 aero-engine disc. From this work, the following conclusions have been drawn:

1. The magnitude and extent of the residual stresses and strains measured using neutron diffraction were shown to be in reasonable agreement with those predicted using a FE quench model.

2. The hoop stresses were generally of the same magnitude as the yield stress in compression on all the disc surfaces. The axial or radial stresses were of magnitude 150 $-200 \mathrm{MPa}$ in compression on the rim / bore and horizontal surfaces, respectively. This is the expected pattern for quenching. Large tensile hoop and radial stresses, up to $400 \mathrm{MPa}$ were observed and predicted within the core of the disc. In the inclined region of the forging, shear and axial stresses of up to $\pm 125 \mathrm{MPa}$ were predicted.

3. FE models provide a virtual capability for the prediction of residual stresses and distortion. However, it is believed that further efforts are required to refine the boundary conditions and input parameters to improve the accuracy of the predictions.

\section{Acknowledgements}

As part of a multi-disciplinary collaborative research program comprised of both industrial and academic partners, the authors would like to acknowledge contributions from Special Metals Wiggin Ltd., WymanGordon Ltd., Rolls-Royce plc, Qinetiq, and the University of Birmingham. The assistance provided by Dr. Daan Maijer at the University of British Columbia with the meshing is gratefully acknowledged. This research was funded in part by the Engineering and Physical Sciences Research Council (GR/N14101).

\section{References}

1. G.A. Webster, Role of residual stress in engineering applications, Materials Science Forum, 347(3) (2000), 1-9.

2. G.A. Webster and A.N. Ezeilo, Residual stress distributions and their influence on fatigue lifetimes, International Journal of Fatigue, 23 (2001), S375-S383.

3. D. Dye, K.T. Conlon and R.C. Reed, Characterisation and modelling of quenchinginduced residual stresses in the nickel-base superalloy IN718, Metallurgical and Materials Transactions A, (2004) in press.

4. A.N. Ezelio, G.A. Webster, P.J. Webster and X. Wang, Characterisation of elastic and plastic deformation in a nickel superalloy using pulsed neutrons, Physica B, 180 (1992), 1044-1046.

5. D. Dye, S.M. Roberts, P.J. Withers and R.C. Reed, The determination of the residual strains and 
stresses in a TIG-welded sheet of IN718 superalloy using neutron diffraction, Journal of Strain Analysis, 35(4) (2000), 247-259.

6. D. Dye, O. Hunziker, S.M. Roberts and R.C. Reed, Modeling of the mechanical effects induced by the tungsten inert gas welding of the IN718 superalloy, Metallurgical and Materials Transactions A, 32A(7) (2001), 1713-1725.

7. Technical Report: High temperature, high strength nickel-base superalloys, Nickel Development Institute, London, 1995.

8. ASM Handbook, vol. 1, Properties and selection: Irons, steels and high performance alloys, $10^{\text {th }} \mathrm{Ed}$., ASM International, Materials Park, OH, 1990.

9. S. Nukiyama, The maximum and minimum values of heat transmitted from metal to boiling water under atmospheric pressure, International Journal of Heat and Mass Transfer, 9 (1966), 1419-1433.

10. J. Goldak, M. Bibby, J. Moore, R. House and B. Patel, Metallurgical and Materials Transactions B, 17B (1986), 587-600.

11. M.P. Jackson and R.C. Reed, Heat treatment of U720Li: The effect of microstructure on properties, Materials Science and Engineering A, 259(1) (1999), 85-97.

12. A.C. Larson and R.B. Von Dreele, General Structure Analysis System (GSAS), Los Alamos National Laboratory Report LAUR 86-748, (2000). 